\title{
MEAN-VALUE PROPERTY ON MANIFOLDS WITH MINIMAL HOROSPHERES
}

\author{
LEONARD TODJIHOUNDE
}

(Received 13 June 2006; accepted 14 June 2007)

Communicated by C. D. Hodgson

\begin{abstract}
Let $(M, g)$ be a non-compact and complete Riemannian manifold with minimal horospheres and infinite injectivity radius. In this paper we prove that bounded functions on $(M, g)$ satisfying the mean-value property are constant. We thus extend a result of Ranjan and Shah ['Harmonic manifolds with minimal horospheres', J. Geom. Anal. 12(4) (2002), 683-694] where they proved a similar result for bounded harmonic functions on harmonic manifolds with minimal horospheres.
\end{abstract}

2000 Mathematics subject classification: 53C21, 53C25.

Keywords and phrases: mean-value property, minimal horospheres.

\section{Introduction}

Let $(M, g)$ be a non-compact and complete Riemannian manifold.

A function $u$ defined on $(M, g)$ is said to have the mean-value property if

$$
\text { for all } r>0 \text { and for all } p \in M, \quad u(p)=\frac{1}{V(p, r)} \int_{B(p, r)} u(q) d \mu(q),
$$

where $d \mu$ denotes the Riemannian volume element and $V(p, r)$ the volume of the closed ball $B(p, r)$ of centre $p$ and radius $r$.

Well-known examples of functions satisfying the mean-value property are harmonic functions on harmonic manifolds (see [9]).

In [6] the authors proved that, on non-compact harmonic manifolds with minimal horospheres, bounded harmonic functions are constant.

One of the major arguments to obtain this result is the fact that, on harmonic manifolds, harmonic functions possess the mean-value property. It thus seems natural

The author thanks warmly the Abdus Salam International Centre for Theoretical Physics for his support during this work.

(C) 2008 Australian Mathematical Society 1446-7887/08 \$A2.00+0.00 
to raise the same question by considering the class of functions satisfying the meanvalue property and defined on manifolds that are not necessarily harmonic.

Some analogous results of Liouville type for functions satisfying the mean-value property have been proved by several authors. For example, the authors in [8] proved that on certain kinds of homogeneous spaces the only $L^{p}$-function satisfying the meanvalue property is the zero function.

For similar results and related works see also $[1-5,10]$ and the references therein.

Our aim is to extend the Liouville type result proved in [6] on bounded functions satisfying the mean-value property and defined on non-compact manifolds with minimal horospheres and infinite injectivity radius.

We refer to [6] and [7] for information and details on the minimality condition of horospheres in a non-compact manifold.

For a real number $r>0$, we consider the stability vector field $H(\cdot, r)$ defined by

$$
H(p, r)=: \int_{B(p, r)} \exp _{p}^{-1}(q) d \mu(q) \text { for all } p \in M
$$

where $\exp _{p}^{-1}$ denotes the inverse of the exponential map.

Let us note that the vanishing of the stability vector field for any radius $r>0$ means that any geodesic ball in $(M, g)$ has its Riemannian centre of mass (or centre of gravity) at the centre of the ball. This is the case for examples of harmonic manifolds, d'Atri spaces or compact locally symmetric spaces.

In the next section we give a result relating the gradient of the volume function and the derivative of the stability vector field that we use in the third section to prove that, on non-compact manifolds with minimal horospheres and infinite injectivity radius, bounded functions having the mean-value property are constant.

\section{Volume functions and stability vector fields}

Let $V:(p, r) \in M \times] 0,+\infty[\longmapsto V(p, r)$ be the function associating to each pair $(p, r) \in M \times] 0,+\infty$ [ the volume $V(p, r)$ of the ball $B(p, r)$.

The volume function $V$ and the stability vector field $H$ are related by the following differential equation.

LEMMA 2.1. Let $\nabla$ denote the gradient operator on $(M, g)$.

For any $r>0$ and $p \in M$, the following holds:

$$
\nabla V(p, r)-\frac{1}{r} \frac{\partial}{\partial r} H(p, r)=0 .
$$

PROOF. For $X \in T_{p} M$, the following holds:

$$
\nabla_{X} V(p, r)=\int_{S(p, r)}\langle\eta(q), X(q)\rangle d \sigma(q),
$$


where $d \sigma$ denotes the Riemannian measure induced on the sphere $S(p, r)$ with centre $p$ and radius $r, \eta(q)$ is the outward unit normal at $q$, and $X(q)$ is the parallel transport of $X$ from $p$ to $q$.

By the Gauss lemma,

$$
\langle\eta(q), X(q)\rangle=\left\langle\left(d \exp _{p}^{-1}\right) \eta(q), X\right\rangle .
$$

Otherwise

$$
\left(d \exp _{p}^{-1}\right) \eta(q)=r^{-1} \exp _{p}^{-1} q
$$

It then follows that

$$
\begin{aligned}
\nabla_{X} V(p, r) & =\int_{S(p, r)} r^{-1} \exp _{p}^{-1} q d \sigma(q) \\
& =r^{-1} \int_{S(p, r)} \exp _{p}^{-1} q d \sigma(q) \\
& =r^{-1} \frac{\partial}{\partial r}\left(\int_{B(p, r)} \exp _{p}^{-1} q d \mu(q)\right) \\
& =r^{-1} \frac{\partial}{\partial r} H(p, r) .
\end{aligned}
$$

Hence the result.

\section{A derivative formula}

Let $p \in M$ and let $X$ be a unit vector in $T_{p} M$. We consider as in [6] the function

$$
\begin{aligned}
\theta_{X}: M-\{p\} & \longrightarrow \mathbb{R} \\
q & \longmapsto \theta_{X}(q)=: L_{p}\left(X, \dot{\gamma}_{q}(0)\right),
\end{aligned}
$$

where $\gamma_{q}$ denotes the geodesic defined by $\gamma_{q}(t)=\exp _{p}\left(t \exp _{p}^{-1} q\right)$, for all $t \in[0,1]$, and $L_{p}\left(X, \dot{\gamma}_{q}(0)\right)$ denotes the angle at $p$ between the vectors $X$ and $\dot{\gamma}_{q}(0)$.

For the geodesic $c$ with $c(0)=p$ and $\dot{c}(0)=X$, let $P_{t}$ be the parallel transport along $c$ and $f_{t}$ the one-parameter family of diffeomorphisms of $M$ given by $f_{t}=\exp _{c(t)} \circ P_{t} \circ \exp _{p}^{-1}$.

Let $u$ be a differentiable function on $M$ possessing the mean-value property.

The following holds.

PROPOSITION 3.1. For any real number $r>0$,

$$
X u(p)=\frac{1}{V(p, r)} \int_{S(p, r)} u \cos \theta_{X} d \sigma-\frac{1}{r} \frac{u(p)}{V(p, r)}\left\langle\frac{\partial}{\partial r} H(p, r), X\right\rangle .
$$


PROOF. Since the function $u$ possesses the mean-value property, we have

$$
u(c(t))=\frac{1}{V(c(t), r)} \int_{B(c(t), r)} u(q) d \mu(q) .
$$

So then

$$
\begin{aligned}
X . u(p)= & \frac{d}{d t} u(c(t))_{\mid t=0} \\
= & \frac{d}{d t}\left(\frac{1}{V(c(t), r)} \int_{B(c(t), r)} u d \mu\right)_{\mid t=0} \\
= & -\frac{1}{V(p, r)^{2}}\langle\nabla V(p, r), X\rangle \int_{B(p, r)} u d \mu \\
& +\frac{1}{V(p, r)} \frac{d}{d t}\left(\int_{B(c(t), r)} u d \mu\right)_{\mid t=0} .
\end{aligned}
$$

From Lemma 2.1,

$$
\nabla V(p, r)=\frac{1}{r} \frac{\partial}{\partial r} H(p, r)
$$

Thus

$$
\begin{aligned}
\frac{1}{V(p, r)^{2}}\langle\nabla V(p, r), X\rangle \int_{B(p, r)} u d \mu & =\frac{1}{V(p, r)^{2}}\left\langle\frac{1}{r} \frac{\partial}{\partial r} H(p, r), X\right\rangle \int_{B(p, r)} u d \mu \\
& =\frac{1}{r} \frac{u(p)}{V(p, r)}\left\langle\frac{\partial}{\partial r} H(p, r), X\right\rangle
\end{aligned}
$$

since

$$
u(p)=\frac{1}{V(p, r)} \int_{B(p, r)} u(q) d \mu(q) .
$$

By Theorem 2.1 in [6] we have

$$
\begin{aligned}
\frac{d}{d t}\left(\int_{B(c(t), r)} u d \mu\right)_{\mid t=0} & =\frac{d}{d t}\left(\int_{B(p, r)} f_{t}^{*}(u d \mu)\right)_{\mid t=0} \\
& =\int_{B(p, r)} \frac{d}{d t}\left(f_{t}^{*}(u d \mu)\right)_{\mid t=0} \\
& =\int_{S(p, r)} u \cos \theta_{X} d \sigma .
\end{aligned}
$$

By replacing (2) and (3) in the relation (1) we obtain the result.

By using the derivative formula given in Proposition 3.1, we get the following.

THEOREM 3.1. Let $(M, g)$ be a non-compact and complete Riemannian manifold with minimal horospheres and infinite injectivity radius.

Any bounded function on $(M, g)$ satisfying the mean-value property is constant. 
PROOF. Let $u$ be a bounded function on $(M, g)$ satisfying the mean-value property.

By Proposition 3.1,

$$
|X u(p)| \leq \alpha \frac{A(p, r)}{V(p, r)}+\frac{\alpha}{V(p, r)}\left\|\frac{1}{r} \frac{\partial}{\partial r} H(p, r)\right\| \quad \text { for all } p \in M \text { and } r>0,
$$

where $A(p, r)$ is the area of the sphere $S(p, r)$, and $\alpha \geq 0$ is such that $|u| \leq \alpha$. But

$$
\begin{aligned}
\left\|\frac{1}{r} \frac{\partial}{\partial r} H(p, r)\right\| & =\left\|\frac{1}{r} \frac{\partial}{\partial r} \int_{B(p, r)} \exp _{p}^{-1} q d \mu(q)\right\| \\
& =\left\|\frac{1}{r} \int_{S(p, r)} \exp _{p}^{-1} q d \sigma(q)\right\| \\
& \leq \frac{1}{r} \int_{S(p, r)}\left\|\exp _{p}^{-1} q\right\| d \sigma(q) \\
& =A(p, r),
\end{aligned}
$$

since $\left\|\exp _{p}^{-1} q\right\|=r$ for all $q \in S(p, r)$.

Thus, we get

$$
|X u(p)| \leq 2 \alpha \frac{A(p, r)}{V(p, r)} .
$$

Due to the minimality of horospheres (see [6] for details),

$$
\lim _{r \rightarrow+\infty} \frac{A(p, r)}{V(p, r)}=K_{\infty}=0 .
$$

By taking the limit of the previous inequality as $r \rightarrow \infty$, it then follows that

$$
|X u(p)|=0 \text { for any } p \in M \text { and any unit vector } X \in T_{p} M .
$$

Hence $u$ is a constant function.

\section{References}

[1] W. Hansen and N. Nadirashvili, 'Liouville's theorem and the restricted mean-value property', J. Math. Pures Appl. (9) 74(2) (1995), 185-198.

[2] D. Heath, 'Functions possessing the mean value properties', Proc. Amer. Math. Soc. 41 (1973), 588-595.

[3] M. El Kadiri, 'Théorème de Liouville et propriété de la moyenne biharmonique restreinte', C. R. Acad. Sci. Paris, Ser. I 1340 (2005), 563-566.

[4] M. El Kadiri, 'Liouville theorem and restricted harmonic mean value property on the real line', Rend. Accad. Naz. Sci. XL Mem. Mat. Appl. (5) 27 (2003), 89-94.

[5] J. Kim and M. W. Wang, 'Invariant mean value property and harmonic functions', Complex Var. Theory Appl. 50(14) (2005), 1049-1059.

[6] A. Ranjan and H. Shah, 'Harmonic manifolds with minimal horospheres', J. Geom. Anal. 12(4) (2002), 683-694.

[7] A. Ranjan and H. Shah, 'Busemann functions in a harmonic manifold', Geom. Dedicata 101 (2003), 167-183. 
[8] A. Sitaram and G. A. Willis, ' $L^{p}$-functions satisfying the mean value property on homogeneous spaces', J. Aust. Math. Soc. Ser. A 56(3) (1994), 384-390.

[9] T. J. Willmore, 'Mean value theorems in harmonic spaces', J. London Math. Soc. 25 (1950), 54 57.

[10] F. Zucca, 'Strong and weak mean value property on trees', Int. J. Pure Appl. Math. 6(1) (2003), $33-49$.

LEONARD TODJIHOUNDE, Institut de Mathematiques et de Sciences Physiques, B.P. 613 Porto-Novo, Republique du Benin

e-mail: leonardt@imsp-uac.org 